\title{
Nonequilibrium first-order phase transition in coupled oscillator systems with inertia and noise
}

\author{
Shamik Gupta ${ }^{1}$, Alessandro Campa ${ }^{2}$ and Stefano Ruffo ${ }^{3}$ \\ ${ }^{1}$ Laboratoire de Physique Théorique et Modèles Statistiques (UMR CNRS 8626), Université Paris-Sud, Orsay, France \\ ${ }^{2}$ Health and Technology Department, Istituto Superiore di Sanità, \\ and INFN Sezione Roma1, Gruppo Collegato Sanità, Roma, Italy \\ ${ }^{3}$ Department of Physics and Astronomy and CSDC, University of Florence, \\ CNISM and INFN, via G. Sansone, 150019 Sesto Fiorentino, Italy
}

\begin{abstract}
We study the dynamics of a system of coupled oscillators of distributed natural frequencies, by including the features of both thermal noise, parametrized by a temperature, and inertial terms, parametrized by a moment of inertia. For a general unimodal frequency distribution, we report here the complete phase diagram of the model in the space of dimensionless moment of inertia, temperature, and width of the frequency distribution. We demonstrate that the system undergoes a nonequilibrium first-order phase transition from a synchronized phase at low parameter values to an incoherent phase at high values. We provide strong numerical evidence for the existence of both the synchronized and the incoherent phase, treating the latter analytically to obtain the corresponding linear stability threshold that bounds the first-order transition point from below. In the limit of zero noise and inertia, when the dynamics reduces to the one of the Kuramoto model, we recover the associated known continuous transition. At finite noise and inertia but in the absence of natural frequencies, the dynamics becomes that of a well-studied model of long-range interactions, the Hamiltonian mean-field model. Close to the first-order phase transition, we show that the escape time out of metastable states scales exponentially with the number of oscillators, which we explain to be stemming from the long-range nature of the interaction between the oscillators.
\end{abstract}

PACS numbers: 05.70.Fh, 05.70.Ln, 05.45.Xt

\section{INTRODUCTION}

Collective synchronization refers to the remarkable phenomenon of a large population of coupled oscillators spontaneously synchronizing to oscillate at a common frequency, despite each constituent having a different natural frequency. This many-body cooperative effect is observed in many physical and biological systems, pervading length and time scales of several orders of magnitude. Some examples are metabolic synchrony in yeast cell suspensions [1], synchronized firings of cardiac pacemaker cells [2], flashing in unison by groups of fireflies [3], voltage oscillations at a common frequency in an array of current-biased Josephson junctions [4], phase synchronization in electrical power distribution networks [5 7], rhythmic applause [8], animal flocking behavior [9]; see Ref. [10] for a recent survey.

A paradigmatic model to study synchronization is the Kuramoto model comprising $N$ phase-only oscillators of distributed natural frequencies that are globally coupled through the sine of their phase differences [11, 12]. Specifically, the system involves $N$ interacting oscillators $i=1,2, \ldots, N$. The $i$-th oscillator has natural frequency $\omega_{i}$, and is characterized by its phase $\theta_{i}$ which is a periodic variable of period $2 \pi$. The $\omega_{i}$ 's have a common probability distribution given by $g(\omega)$. The phase $\theta_{i}$ evolves in time according to the equation

$$
\frac{\mathrm{d} \theta_{i}}{\mathrm{~d} t}=\omega_{i}+\frac{\widetilde{K}}{N} \sum_{j=1}^{N} \sin \left(\theta_{j}-\theta_{i}\right),
$$

where $\widetilde{K}$ is the coupling constant, while the factor $1 / N$ makes the model well behaved in the continuum limit $N \rightarrow \infty$.

In this work, we study a generalization of the dynamics Eq. (1) that includes inertial terms parametrized by a moment of inertia and stochastic noise parametrized by a temperature [13 15]. Noise accounts for the temporal fluctuations of the natural frequencies [16], while inertia elevates the first-order Kuramoto dynamics to secondorder [17]. For a general unimodal distribution of the natural frequencies, we report here the complete phase diagram of the model in the space of dimensionless moment of inertia, temperature, and width of the frequency distribution, showing that the system in the steady state may exist in either of two possible phases, namely, a synchronized phase and an unsynchronized or incoherent phase. We show that a nonequilibrium first-order transition occurs from the synchronized phase at low parameter values to the incoherent phase at high values. While strong numerical evidence is provided to support the existence of both the synchronized and the incoherent phase, only the latter could be treated analytically to obtain the corresponding linear stability threshold that bounds the first-order transition point from below. In proper limits of the dynamics, we recover the known continuous phase transitions in the Kuramoto model and in its noisy extension [16], and an equilibrium continuous transition in a related model of long-range interactions, the Hamiltonian mean-field model [18].

The Kuramoto model has been almost exclusively studied within the field of synchronization and non-linear 
dynamical systems. On the other hand, there has been much recent activity within the community of statistical physicists to study nonequilibrium stationary states (NESSs) and develop a general framework akin to the one due to Boltzmann and Gibbs for equilibrium that allows analysis of nonequilibrium states on a general footing [19]. Unfortunately, there are few examples of NESSs for which one knows the probability measure of configurations exactly, so that the bulk of studies have relied on numerical simulations and approximate analysis [20].

Our work interprets the dynamics of the Kuramoto model to be of true non-equilibrium character. Moreover, quenched disorder in the form of natural frequencies of the oscillators provides a very rich setting to study the interplay of the nonequilibrium character of the dynamics with the disorder. In this rich backdrop, we are able to characterize the nature of the NESS and ascertain under quite general conditions the whole spectrum of phase transitions.

The paper is organized as follows. In the following section, we describe the model of interest and briefly review previous studies of the model. In Sec. III, we present the complete phase diagram of the model, providing numerical simulation results in support. In Sec. IV we present an analytical treatment of the properties of the incoherent phase, based on the Kramers equation for the single-oscillator distribution. This is followed in Sec. V by a comparison of our analytical predictions with numerical simulations. The paper ends with conclusions. Some of the technical details are relegated to the two appendices.

\section{THE MODEL}

We now give a precise definition of the generalized dynamics that we study in this paper. In addition to phase $\theta_{i}$, we associate with the $i$-th oscillator another dynamical variable, namely, the angular velocity $v_{i}$. With a Gaussian noise force $\eta_{i}(t)$ and the natural frequency $\omega_{i}$, the dynamics is [13, 15]

$\frac{\mathrm{d} \theta_{i}}{\mathrm{~d} t}=v_{i}, m \frac{\mathrm{d} v_{i}}{\mathrm{~d} t}=-\gamma v_{i}+K r \sin \left(\psi-\theta_{i}\right)+\gamma \omega_{i}+\sqrt{\gamma} \eta_{i}(t)$,

where $m$ is the oscillator moment of inertia, $\gamma$ is the friction constant, while $r$ is the synchronization order parameter:

$$
r(t) e^{i \psi(t)} \equiv \frac{\sum_{j=1}^{N} e^{i \theta_{j}(t)}}{N}
$$

Here, we have

$$
\left\langle\eta_{i}(t)\right\rangle=0,\left\langle\eta_{i}(t) \eta_{j}\left(t^{\prime}\right)\right\rangle=2 T \delta_{i j} \delta\left(t-t^{\prime}\right),
$$

with temperature $T$ in units of the Boltzmann constant. We consider a unimodal $g(\omega)$ (that is, symmetric about mean $\widetilde{\omega}$, and decreases to zero with increasing $|\omega-\widetilde{\omega}|)$, and denote its width by $\sigma$. In the absence of inertia, the dynamics with the redefinition $K / \gamma=\widetilde{K}$ reduces at $T=0$ to that of the Kuramoto model [11, 12] and at $T \neq 0$ to that of its extension studied by Sakaguchi in Ref. 16].

The dynamics Eq. (2) also describes motion of particles with an $X Y$-interaction on a unit circle, with $\theta_{i}, v_{i}$ and $\gamma \omega_{i}$ being respectively the angular coordinate, velocity and external torque. In the absence of $\omega_{i}$ 's, Eq. (2) for $\gamma=0$ is the microcanonical dynamics of the Hamiltonian mean-field model [18], a prototype of long-range interacting systems 21]. In this case, the equations of motion are the Hamilton equations associated with the Hamiltonian

$$
H=\sum_{i=1}^{N} \frac{p_{i}^{2}}{2 m}+\frac{K}{2 N} \sum_{i, j=1}^{N}\left[1-\cos \left(\theta_{i}-\theta_{j}\right)\right],
$$

with $p_{i}=m v_{i}$ the momentum of the $i$-th particle. The dynamics of this system is microcanonical, conserving energy and total momentum. With no $\omega_{i}$ 's, but $\gamma \neq 0$, the dynamics of the resulting Brownian mean-field (BMF) model is canonical, mimicking the interaction of the HMF system with a heat bath [22].

The dynamics Eq. (2) is invariant under $\theta_{i} \rightarrow \theta_{i}+$ $\widetilde{\omega} t, v_{i} \rightarrow v_{i}+\widetilde{\omega}, \omega_{i} \rightarrow \omega_{i}+\widetilde{\omega}$, and the effect of $\sigma$ may be made explicit by replacing $\omega_{i}$ in the second equation with $\sigma \omega_{i}$. We thus consider from now on the dynamics Eq. (2) with the substitution $\omega_{i} \rightarrow \sigma \omega_{i}$. In the resulting model, we take $g(\omega)$ to have zero mean and unit width, without loss of generality.

For $m \neq 0$, using dimensionless variables

$$
\begin{aligned}
& \bar{t} \equiv t \sqrt{K / m}, \\
& \bar{v}_{i} \equiv v_{i} \sqrt{m / K}, \\
& 1 / \sqrt{\bar{m}} \equiv \gamma / \sqrt{K m}, \\
& \bar{\sigma} \equiv \gamma \sigma / K, \\
& \bar{T} \equiv T / K, \\
& \bar{\eta}_{i}(\bar{t}) \equiv \eta_{i}(t) \sqrt{\gamma} / K,
\end{aligned}
$$

the dynamics becomes

$$
\frac{\mathrm{d} \theta_{i}}{\mathrm{~d} \bar{t}}=\bar{v}_{i}, \frac{\mathrm{d} \bar{v}_{i}}{\mathrm{~d} \bar{t}}=-\frac{1}{\sqrt{\bar{m}}} \bar{v}_{i}+r \sin \left(\psi-\theta_{i}\right)+\bar{\sigma} \omega_{i}+\bar{\eta}_{i}(\bar{t}),
$$

where

$$
\left\langle\bar{\eta}_{i}(\bar{t}) \bar{\eta}_{j}\left(\bar{t}^{\prime}\right)\right\rangle=2 \frac{\bar{T}}{\sqrt{\bar{m}}} \delta_{i j} \delta\left(\bar{t}-\bar{t}^{\prime}\right) .
$$

For $m=0$, using dimensionless time $\bar{t} \equiv t(K / \gamma)$, the dynamics becomes the overdamped motion

$$
\frac{\mathrm{d} \theta_{i}}{\mathrm{~d} \bar{t}}=r \sin \left(\psi-\theta_{i}\right)+\bar{\sigma} \omega_{i}+\bar{\eta}_{i}(\bar{t}),
$$

where

$$
\left\langle\bar{\eta}_{i}(\bar{t}) \bar{\eta}_{j}\left(\bar{t}^{\prime}\right)\right\rangle=2 \bar{T} \delta_{i j} \delta\left(\bar{t}-\bar{t}^{\prime}\right)
$$


From now on, we will consider in place of dynamics Eq. (2) the reduced dynamics Eq. (12) [that reduces for $m=0$ to the overdamped dynamics Eq. (14)] involving three dimensionless parameters, $\bar{m}, \bar{T}$, $\bar{\sigma}$; we will drop overbars for simplicity of notation. With $\sigma=0$ (i.e. $g(\omega)=\delta(\omega)[13],[15])$, the resulting BMF dynamics has an equilibrium stationary state [22]. For other $g(\omega)$, the dynamics Eq. (12) violates detailed balance due to the external driving by the set of torques $\left\{\gamma \omega_{i}\right\}$, yielding a NESS. We demonstrate this in Appendix A.

Several stationary state aspects of the dynamics Eq. (12) in the continuum limit $N \rightarrow \infty$ are known. For the Kuramoto dynamics $(m=T=0)$, the system exhibits a continuous transition from a low- $\sigma$ synchronized $\left[r_{\mathrm{st}}=\right.$ $r(t \rightarrow \infty) \neq 0]$ to a high- $\sigma$ incoherent $\left(r_{\mathrm{st}}=0\right)$ phase across the critical point [11]

$$
\sigma_{c}(m=0, T=0)=\frac{\pi g(0)}{2} ;
$$

extending to $T \neq 0$, the point becomes a second-order critical line $\sigma_{c}(m=0, T)$ on the $(T, \sigma)$-plane, given, on using the results of Sakaguchi in Ref. [16], by solving

$$
2=\int_{-\infty}^{\infty} \frac{T g(\omega) \mathrm{d} \omega}{T^{2}+\omega^{2} \sigma_{c}^{2}(m=0, T)} .
$$

For the BMF dynamics $(\sigma=0 ; m, T \neq 0)$, the synchronization transition is again continuous, occurring at the critical temperature given by 22]

$$
T_{c}=\frac{1}{2} \text {. }
$$

Although there have been some numerical studies of the full dynamics for non-zero $m, T, \sigma$ [13, 15, 23], the complete synchronization phase diagram for a general unimodal $g(\omega)$ has not been addressed before, a question we take up and answer in this paper. In the next section, we describe the complete phase diagram that emerges out of our analysis.

\section{PHASE DIAGRAM}

The complete phase diagram is shown schematically in Fig. 1(a), where the thick red second-order critical lines stand for the continuous transitions mentioned above. For non-zero $m, T, \sigma$, the synchronization transition becomes first-order, occurring across the shaded blue transition surface; this surface is bounded by the second-order critical lines on the $(T, \sigma)$ and $(m, T)$ planes, and by a first-order transition line on the $(m, \sigma)$-plane.

The phase diagram in Fig. 1(a) is a generalization of the one for typical fluids where a first-order transition line ends in a critical point, while we have here a first-order transition surface ending in critical lines. All transitions for $\sigma \neq 0$ are in NESS, and we interpret them to be of dynamical origin, accounted for by stability considerations of stationary solutions of equations describing evolution
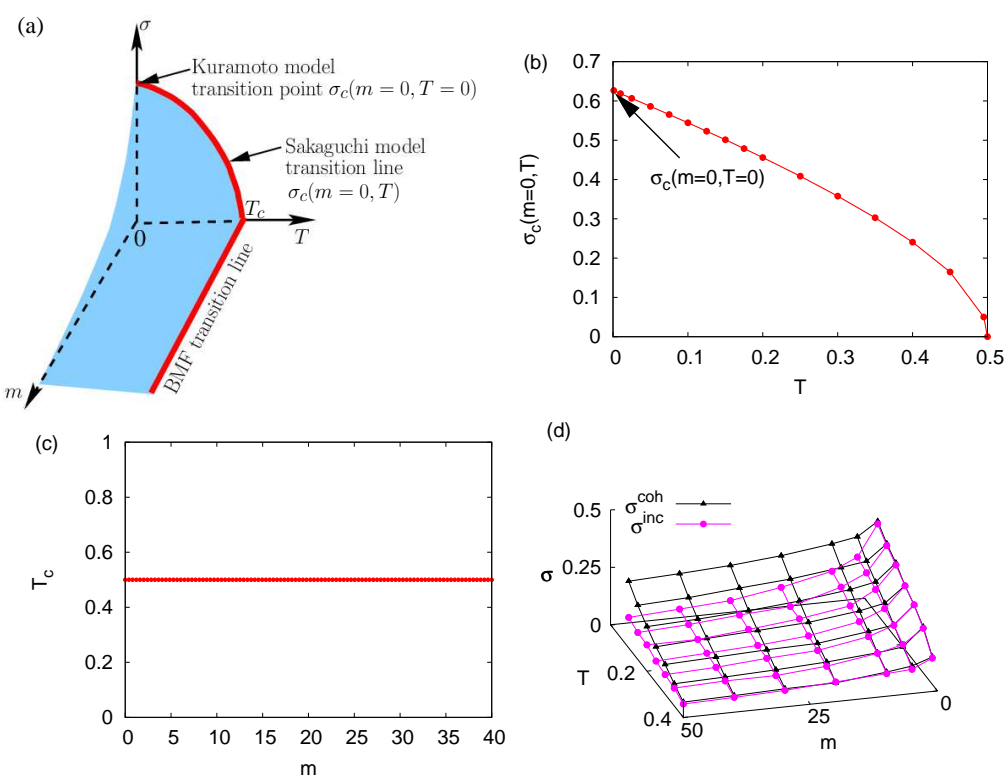

(d)
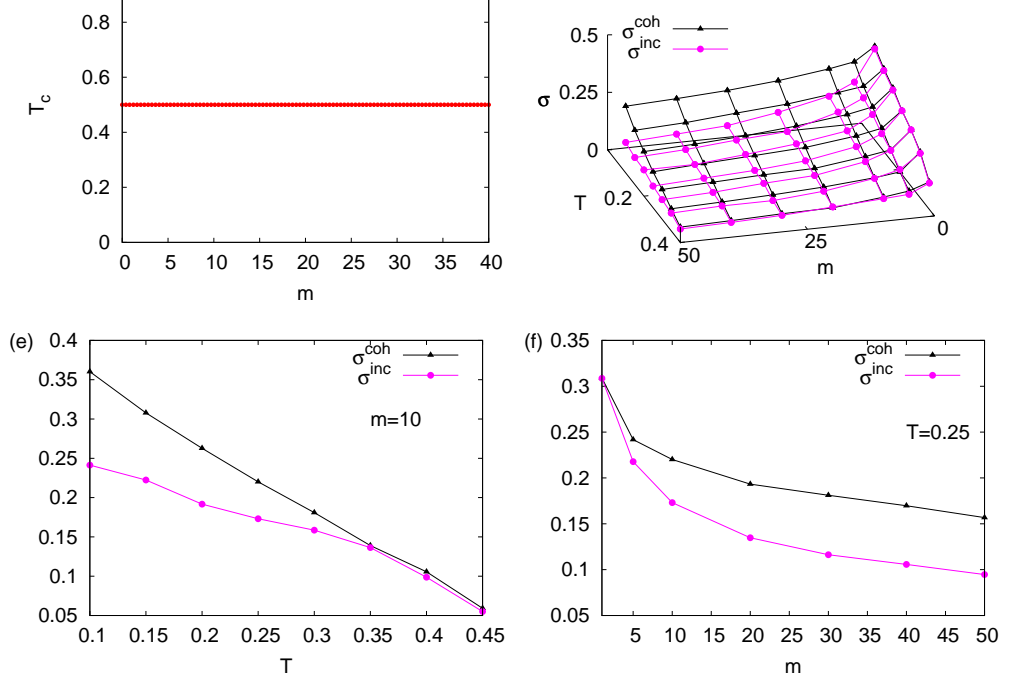

FIG. 1. (Color online) (a) Schematic phase diagram of model Eq. (12) in terms of dimensionless moment of inertia $m$, temperature $T$, and width $\sigma$ of the frequency distribution: the shaded blue surface is a first-order transition surface, the thick red lines are second-order critical lines. The system is synchronized inside the region bounded by the surface, and is incoherent outside. The limits (the Kuramoto model, the Sakaguchi model and the BMF model) in which known transitions are obtained are labeled. (b) The known transition line for the Sakaguchi model, given by Eq. (17), showing also the Kuramoto model transition point, Eq. (16), for a Gaussian $g(\omega)$ with zero mean and unit width 24]. (c) The known transition line for the BMF model, given by Eq. (18). The shaded blue surface in (a) is bounded from above and below by the dynamical stability thresholds $\sigma^{\mathrm{coh}}(m, T)$ and $\sigma^{\text {inc }}(m, T)$ of the synchronized and the incoherent phase respectively. These thresholds may be estimated in $N$-body simulations from hysteresis plots (see Fig. 2 for an example); Panel (d) shows the surfaces $\sigma^{\text {coh }}(m, T)$ and $\sigma^{\text {inc }}(m, T)$ obtained from $N$-body simulations with $N=500$ for a Gaussian $g(\omega)$ with zero mean and unit width, with cuts of the three-dimensional plot at $m=10$ shown in panel (e) and at $T=0.25$ shown in panel (f).

of phase-space distribution. Showing that the phases extremize a free-energy-like quantity (e.g., a large deviation functional [25]) in NESS is a daunting task in the absence of a general framework akin to that for equilibrium [26]. For $\sigma=0$, the different phases actually minimize the 


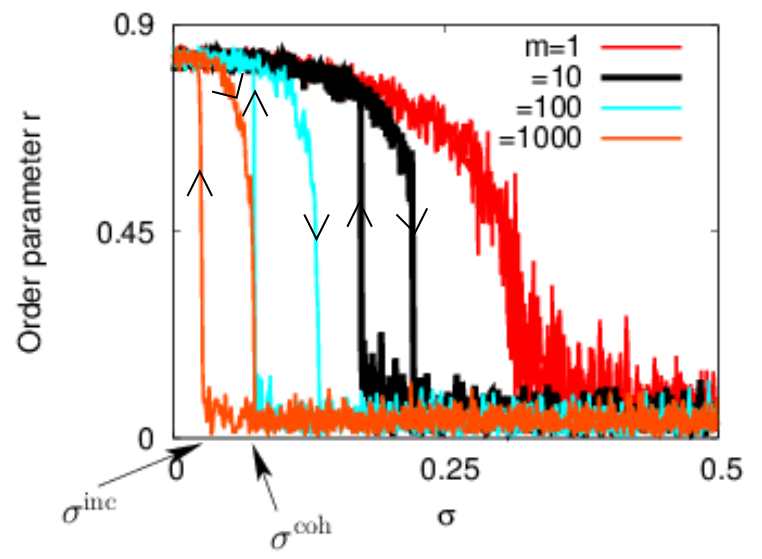

FIG. 2. (Color online) $r$ vs. adiabatically tuned $\sigma$ for different $m$ values at $T=0.25<T_{c}=1 / 2$, showing also the stability thresholds, $\sigma^{\text {inc }}(m, T)$ and $\sigma^{\text {coh }}(m, T)$, for $m=1000$. For a given $m$, the branch of the plot to the right (left) marked with an arrowhead pointing down (up) corresponds to $\sigma$ increasing (decreasing); for $m=1$, the two branches almost overlap. The data are obtained in $N$-body simulations with $N=500$ for a Gaussian $g(\omega)$ with zero mean and unit width.

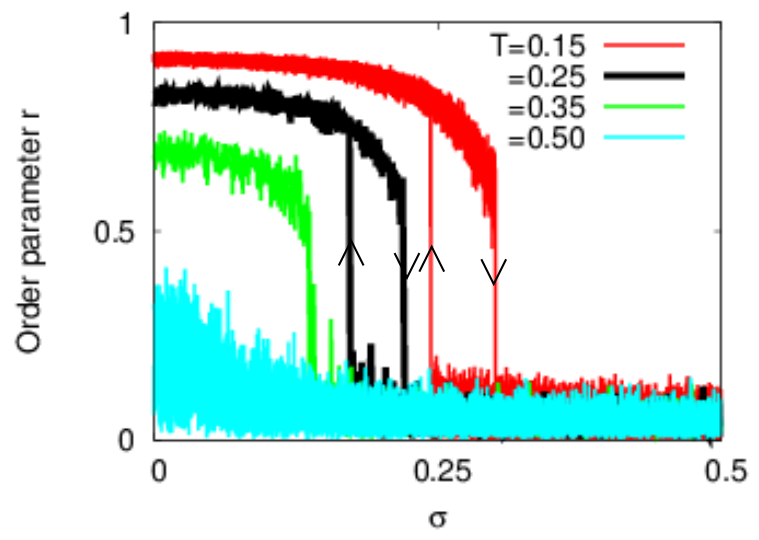

FIG. 3. (Color online) $r$ vs. adiabatically tuned $\sigma$ for different temperatures $T \leq T_{c}=1 / 2$ at a fixed moment of inertia $m=10$. For a given $T$, the branch of the plot to the right (left) marked with an arrowhead pointing down (up) corresponds to $\sigma$ increasing (decreasing); for $T \geq 0.35$, the two branches almost overlap. The data are obtained in $N$-body simulations with $N=500$ for a Gaussian $g(\omega)$ with zero mean and unit width. Similar disappearance of the hysteresis loop with increase of $T$ was reported in Ref. [14].

equilibrium free energy [21].

To confirm the first-order transition, we performed $N$ body simulations involving integrations of Eq. (12) for a representative $g(\omega)$, i.e., a Gaussian. Details of the simulation procedure are given in the Appendix B. For given $m$ and $T$ and an initial state with oscillators at $\theta=0$ and angular velocities sampled from a Gaussian distribution with zero mean and width $\propto T$, we let the system equilibrate at $\sigma=0$. We then tune $\sigma$ adiabati-

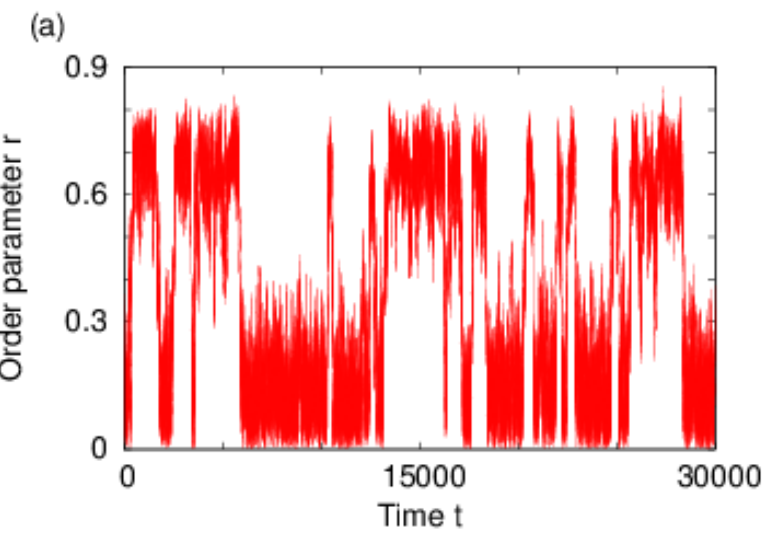

(b)

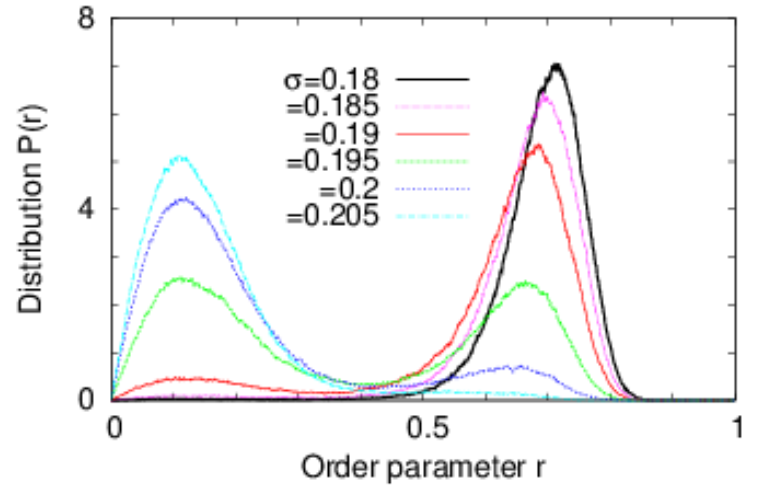

FIG. 4. (Color online) For $m=20, T=0.25$, and a Gaussian $g(\omega)$ with zero mean and unit width, (a) shows at $\sigma=0.195$, the numerically estimated first-order phase transition point, $r$ vs. time in the stationary state, while (b) shows the distribution $P(r)$ at several $\sigma$ 's around 0.195 . The data are obtained in $N$-body simulations with $N=100$.

cally to high values and back in a cycle. Figure 2 shows the behavior of the synchronization order parameter $r$ for several $m$ 's at a fixed $T$ less than the BMF transition point $T_{c}=1 / 2$, illustrating sharp jumps and hysteresis behavior expected of a first-order transition. With decrease of $m$, the jump in $r$ becomes less sharp and the hysteresis loop area decreases, both consistent with the transition becoming second-order-like as $m$ decreases, see Fig. 1. For $m=1000$, Fig. 2] shows $\sigma^{\text {inc }}(m, T)$ and $\sigma^{\operatorname{coh}}(m, T)$, the stability thresholds for the incoherent and the synchronized phase, respectively; the phase transition point $\sigma_{c}(m, T)$ lies in between the two thresholds (see Fig. 1(d)). Figure 2 shows that the thresholds decrease and approach zero with the increase of $m$; it also suggests, together with Fig. 3, that $\sigma^{\text {inc }}$ and $\sigma^{\text {coh }}$ coincide both on the second order critical lines and as $m \rightarrow \infty$ at a fixed $T$.

For given $m$ and $T$ and $\sigma$ between $\sigma^{\text {inc }}(m, T)$ and $\sigma^{\mathrm{coh}}(m, T), r$ versus time in the stationary state shows bistability, with the system switching back and forth between incoherent and synchronized states [Fig. 4(a)]. To have not-too-large switching times, these simulations 
have been performed with a relatively small number of oscillators, $N=100$, causing large fluctuations in the order parameter $r$. Therefore, in Fig. 4(a) the synchronized and the unsynchronized state are characterized by values of $r$ fluctuating above and below 0.4 , respectively; however, this does allow for a clear visualization of the switches. The distribution $P(r)$ in Fig. 4(b) is bimodal with a peak around $r \approx 0$ or $r>0$ as $\sigma$ varies between $\sigma^{\text {inc }}$ and $\sigma^{\text {coh }}$, consistent with the transition being firstorder. Indeed, a first-order transition point is characterized by two equally likely values of the order parameter, while at a second-order phase transition point, the order parameter has its value equal to zero [27].

\section{ANALYTICAL TREATMENT}

We now turn to an analytical treatment of the firstorder transition. In the continuum limit $N \rightarrow \infty$, the dynamics Eq. (12) is described by the single-oscillator distribution $f(\theta, v, \omega, t)$ which gives at time $t$ and for each $\omega$ the fraction of oscillators with phase $\theta$ and angular velocity $v$. The distribution is $2 \pi$-periodic in $\theta$, and obeys the normalization $\int_{0}^{2 \pi} \mathrm{d} \theta \int_{-\infty}^{\infty} \mathrm{d} v f(\theta, v, \omega, t)=1$, while evolving following the Kramers equation [15]

$\frac{\partial f}{\partial t}=-v \frac{\partial f}{\partial \theta}+\frac{\partial}{\partial v}\left(\frac{v}{\sqrt{m}}-\sigma \omega-r \sin (\psi-\theta)\right) f+\frac{T}{\sqrt{m}} \frac{\partial^{2} f}{\partial v^{2}}$

where $r e^{i \psi}=\int \mathrm{d} \theta \mathrm{d} v \mathrm{~d} \omega g(\omega) e^{i \theta} f(\theta, v, \omega, t)$. We now give the derivation of Eq. (19), followed by a discussion of its stationary solution corresponding to the incoherent phase.

\section{A. The Kramers equation for the single-oscillator distribution: Incoherent stationary state}

Here, we start with deriving the Bogoliubov-BornGreen-Kirkwood-Yvon (BBGKY) hierarchy equations for the dynamics Eq. (12) for any number of oscillators $N$, and, from there, by considering the limit $N \rightarrow \infty$, obtain the Kramers Eq. (19). For simplicity, we first discuss the derivation of the BBGKY equations for the case of a bimodal $g(\omega)$, and then generalize it to a general $g(\omega)$.

Consider a given realization of $g(\omega)$, in which there are $N_{1}$ oscillators with frequencies $\omega_{1}$, and $N_{2}$ oscillators with frequencies $\omega_{2}$, where $N_{1}+N_{2}=N$. We then define the $N$-oscillator distribution function $f_{N}\left(\theta_{1}, v_{1}, \ldots, \theta_{N_{1}}, v_{N_{1}}, \theta_{N_{1}+1}, v_{N_{1}+1}, \ldots, \theta_{N}, v_{N}, t\right)$ as the probability density at time $t$ to observe the system around the values $\left\{\theta_{i}, v_{i}\right\}_{1 \leq i \leq N}$. In the following, we use the shorthand notations $z_{i} \equiv\left(\theta_{i}, v_{i}\right)$ and $\mathbf{z}=\left(z_{1}, z_{2}, \ldots, z_{N}\right)$. Note that $f_{N}$ satisfies the normalization $\int\left(\prod_{i=1}^{N} \mathrm{~d} z_{i}\right) f_{N}(\mathbf{z}, t)=1$. We assume that
1. $f_{N}$ is symmetric with respect to permutations of dynamical variables within the group of oscillators with the same frequency, and

2. $f_{N}$, together with the derivatives $\partial f_{N} / \partial v_{i} \forall i$, vanish on the boundaries of the phase space.

The evolution of $f_{N}$ follows the Fokker-Planck equation which may be straightforwardly derived from the equations of motion Eq. (12):

$$
\begin{aligned}
& \frac{\partial f_{N}}{\partial t}=-\sum_{i=1}^{N}\left[v_{i} \frac{\partial f_{N}}{\partial \theta_{i}}-\frac{1}{\sqrt{m}} \frac{\partial\left(v_{i} f_{N}\right)}{\partial v_{i}}\right] \\
& -\sigma \sum_{j=1}^{N}\left(\Omega^{T}\right)_{j} \frac{\partial f_{N}}{\partial v_{j}}+\frac{T}{\sqrt{m}} \sum_{i=1}^{N} \frac{\partial^{2} f_{N}}{\partial v_{i}^{2}} \\
& -\frac{1}{2 N} \sum_{i, j=1}^{N} \sin \left(\theta_{j}-\theta_{i}\right)\left[\frac{\partial f_{N}}{\partial v_{i}}-\frac{\partial f_{N}}{\partial v_{j}}\right]
\end{aligned}
$$

where we have defined the $N \times 1$ column vector $\Omega$ whose first $N_{1}$ entries equal $\omega_{1}$ and the following $N_{2}$ entries equal $\omega_{2}$, and where the superscript $T$ denotes matrix transpose operation: $\Omega^{T} \equiv\left[\begin{array}{lllll}\omega_{1} & \omega_{1} \ldots \omega_{1} & \omega_{2} & \ldots & \omega_{2}\end{array}\right]$.

To proceed, we follow standard procedure [28], and define the reduced distribution function $f_{s_{1}, s_{2}}$, with $s_{1}=$ $0,1,2, \ldots, N_{1}$ and $s_{2}=0,1,2, \ldots, N_{2}$, as

$$
\begin{aligned}
& f_{s_{1}, s_{2}}\left(z_{1}, z_{2}, \ldots, z_{s_{1}}, z_{N_{1}+1}, \ldots, z_{N_{1}+s_{2}}, t\right) \\
& =\frac{N_{1} !}{\left(N_{1}-s_{1}\right) ! N_{1}^{s_{1}}} \frac{N_{2} !}{\left(N_{2}-s_{2}\right) ! N_{2}^{s_{2}}} \\
& \int \mathrm{d} z_{s_{1}+1} \ldots \mathrm{d} z_{N_{1}} \mathrm{~d} z_{N_{1}+s_{2}+1} \ldots \mathrm{d} z_{N} f_{N}(z, t) .
\end{aligned}
$$

Note that the following normalizations hold for the single-oscillator distribution functions: $\int \mathrm{d} z_{1} f_{1,0}\left(z_{1}, t\right)=$ 1 , and $\int \mathrm{d} z_{N_{1}+1} f_{0,1}\left(z_{N_{1}+1}, t\right)=1$.

Using Eq. (20) in Eq. (21) and simplifying, we get the BBGKY hierarchy equations for oscillators with frequencies $\omega_{1}$ as

$$
\begin{aligned}
& \frac{\partial f_{s, 0}}{\partial t}+\sum_{i=1}^{s}\left[\frac{v_{i} \partial f_{s, 0}}{\partial \theta_{i}}-\frac{1}{\sqrt{m}} \frac{\partial}{\partial v_{i}}\left(v_{i} f_{s, 0}\right)\right]+\sigma \sum_{i=1}^{s} \omega_{1} \frac{\partial f_{s, 0}}{\partial v_{i}} \\
& -\frac{T}{\sqrt{m}} \sum_{i=1}^{s} \frac{\partial^{2} f_{s, 0}}{\partial v_{i}^{2}}=-\frac{1}{2 N} \sum_{i, j=1}^{s} \sin \left(\theta_{j}-\theta_{i}\right)\left[\frac{\partial f_{s, 0}}{\partial v_{i}}-\frac{\partial f_{s, 0}}{\partial v_{j}}\right] \\
& -\frac{N_{1}}{N} \sum_{i=1}^{s} \int \mathrm{d} z_{s+1} \sin \left(\theta_{s+1}-\theta_{i}\right) \frac{\partial f_{s+1,0}}{\partial v_{i}} \\
& -\frac{N_{2}}{N} \int \mathrm{d} z_{N_{1}+1} \sum_{i=1}^{s} \sin \left(\theta_{N_{1}+1}-\theta_{i}\right) \frac{\partial f_{s, 1}}{\partial v_{i}}
\end{aligned}
$$

and similar equations for $f_{0, s}$ for oscillators of frequencies 
$\omega_{2}$. The first equations of the hierarchy are

$$
\begin{aligned}
& \frac{\partial f_{1,0}(\theta, v, t)}{\partial t}+\frac{v \partial f_{1,0}(\theta, v, t)}{\partial \theta}-\frac{1}{\sqrt{m}} \frac{\partial}{\partial v}\left(v f_{1,0}(\theta, v, t)\right) \\
& +\sigma \omega_{1} \frac{\partial f_{1,0}(\theta, v, t)}{\partial v}-\frac{T}{\sqrt{m}} \frac{\partial^{2} f_{1,0}(\theta, v, t)}{\partial v^{2}} \\
& =-\frac{N_{1}}{N} \int \mathrm{d} \theta^{\prime} \mathrm{d} v^{\prime} \sin \left(\theta^{\prime}-\theta\right) \frac{\partial f_{2,0}\left(\theta, v, \theta^{\prime}, v^{\prime}, t\right)}{\partial v} \\
& -\frac{N_{2}}{N} \int \mathrm{d} \theta^{\prime} \mathrm{d} v^{\prime} \sin \left(\theta^{\prime}-\theta\right) \frac{\partial f_{1,1}\left(\theta, v, \theta^{\prime}, v^{\prime}, t\right)}{\partial v}
\end{aligned}
$$

and

$$
\begin{aligned}
& \frac{\partial f_{0,1}(\theta, v, t)}{\partial t}+\frac{v \partial f_{0,1}(\theta, v, t)}{\partial \theta}-\frac{1}{\sqrt{m}} \frac{\partial}{\partial v}\left(v f_{0,1}(\theta, v, t)\right) \\
& +\sigma \omega_{2} \frac{\partial f_{0,1}(\theta, v, t)}{\partial v}-\frac{T}{\sqrt{m}} \frac{\partial^{2} f_{0,1}(\theta, v, t)}{\partial v^{2}} \\
& =-\frac{N_{2}}{N} \int \mathrm{d} \theta^{\prime} \mathrm{d} v^{\prime} \sin \left(\theta^{\prime}-\theta\right) \frac{\partial f_{0,2}\left(\theta, v, \theta^{\prime}, v^{\prime}, t\right)}{\partial v} \\
& -\frac{N_{1}}{N} \int \mathrm{d} \theta^{\prime} \mathrm{d} v^{\prime} \sin \left(\theta^{\prime}-\theta\right) \frac{\partial f_{1,1}\left(\theta, v, \theta^{\prime}, v^{\prime}, t\right)}{\partial v}
\end{aligned}
$$

In the limit of large $N$, we can write

$$
g(\omega)=\left[\frac{N_{1}}{N} \delta\left(\omega-\omega_{1}\right)+\frac{N_{2}}{N} \delta\left(\omega-\omega_{2}\right)\right],
$$

and express Eqs. (23) and (24) in terms of $g(\omega)$.

In order to generalize Eqs. (23) and (24) to the case of a continuous $g(\omega)$, we denote for this case the singleoscillator distribution function as $f(\theta, v ; \omega, t)$. The first equation of the hierarchy is then

$$
\begin{aligned}
& \frac{\partial f(\theta, v, \omega, t)}{\partial t}+\frac{v \partial f(\theta, v, \omega, t)}{\partial \theta}-\frac{1}{\sqrt{m}} \frac{\partial}{\partial v}(v f(\theta, v, \omega, t)) \\
& +\sigma \omega \frac{\partial f(\theta, v, \omega, t)}{\partial v}-\frac{T}{\sqrt{m}} \frac{\partial^{2} f(\theta, v, \omega, t)}{\partial v^{2}} \\
& =-\int \mathrm{d} \omega^{\prime} g\left(\omega^{\prime}\right) \int \mathrm{d} \theta^{\prime} \mathrm{d} v^{\prime} \sin \left(\theta^{\prime}-\theta\right) \frac{\partial f\left(\theta, v, \theta^{\prime}, v^{\prime}, \omega, \omega^{\prime}, t\right)}{\partial v} .
\end{aligned}
$$

In the continuum limit $N \rightarrow \infty$, we may neglect two-oscillator correlations and approximate $f\left(\theta, v, \theta^{\prime}, v^{\prime}, \omega, \omega^{\prime}, t\right)$ as

$$
\begin{aligned}
& f\left(\theta, v, \theta^{\prime}, v^{\prime}, \omega, \omega^{\prime}, t\right)=f(\theta, v, \omega, t) f\left(\theta^{\prime}, v^{\prime}, \omega^{\prime}, t\right) \\
& + \text { corrections subdominant in } N,
\end{aligned}
$$

so that Eq. (26) reduces to the Kramers Eq. (19).

The stationary solutions of Eq. (19) are obtained by setting the left hand side to zero. For $\sigma=0$, the stationary solution is

$$
f_{\mathrm{st}}(\theta, v) \propto \exp \left[-\left(v^{2} / 2-r_{\mathrm{st}} \cos \theta\right) / T\right],
$$

that corresponds to canonical equilibrium, with $r_{\text {st }}$ determined self-consistently [22]. For $\sigma \neq 0$, the incoherent stationary state is 15

$$
f_{\mathrm{st}}^{\mathrm{inc}}(\theta, v, \omega)=\frac{1}{(2 \pi)^{3 / 2} \sqrt{T}} \exp \left[-(v-\sigma \omega \sqrt{m})^{2} /(2 T)\right] .
$$

The existence of the synchronized stationary state is borne out by our simulation results discussed above (see Figs. 213, and 4), although its analytical form is not known.

\section{B. Linear stability analysis of the incoherent state}

Let us now discuss the linear stability analysis of the incoherent state Eq. (29), pursued in Ref. [15] by linearizing Eq. (19) about the state by expanding $f$ as

$$
f(\theta, v, \omega, t)=f_{\mathrm{st}}^{\mathrm{inc}}(\theta, v, \omega)+e^{\lambda t} \delta f(\theta, v, \omega),
$$

with $\delta f \ll 1$. The solution of the linearized equation yields that $\lambda$ satisfies [15]

$$
\frac{2 T}{e^{m T}}=\sum_{p=0}^{\infty} \frac{(-m T)^{p}\left(1+\frac{p}{m T}\right)}{p !} \int_{-\infty}^{\infty} \frac{g(\omega) \mathrm{d} \omega}{1+\frac{p}{m T}+i \frac{\sigma \omega}{T}+\frac{\lambda}{T \sqrt{m}}} .
$$

The above equation contains valuable information about the range of values of the parameters $m, T, \sigma$ for which the incoherent state is stable, and consequently, about the transition from the incoherent to synchronized phase. This warrants a detailed analysis of Eq. (31) for a general unimodal $g(\omega)$. The analysis for Lorentzian $g(\omega)$ in Ref. [15] left untouched the crucial issue of the synchronization transition.

We rewrite Eq. (31) as

$$
\begin{aligned}
& F(\lambda ; m, T, \sigma) \equiv \frac{e^{m T}}{2 T} \sum_{p=0}^{\infty} \frac{(-m T)^{p}\left(1+\frac{p}{m T}\right)}{p !} \\
& \times \int \frac{g(\omega) \mathrm{d} \omega}{1+\frac{p}{m T}+\frac{\lambda}{T \sqrt{m}}+i \frac{\sigma \omega}{T}}-1=0,
\end{aligned}
$$

where $g(\omega)$ is unimodal. The incoherent state is unstable if there is a $\lambda$ with a positive real part that satisfies the above eigenvalue equation. We will now prove that, depending on the values of the parameters appearing in the above equation, there can be at most one such $\lambda$ that can be only real. In addition, for the case of a Gaussian $g(\omega)$ explicitly used in simulations reported in this paper, we obtain the general shape of the surface in the $(m, T, \sigma)$ space that defines the instability region of the incoherent state.

Considering $m$ and $T$ strictly positive, we multiply for convenience the numerator and denominator of Eq. (32) by $m T$ to obtain

$$
\begin{aligned}
& F(\lambda ; m, T, \sigma)=\frac{e^{m T}}{2 T} \sum_{p=0}^{\infty} \frac{(-m T)^{p}(p+m T)}{p !} \\
& \times \frac{g(\omega) \mathrm{d} \omega}{m T+p+\sqrt{m} \lambda+i \sigma m \omega}-1=0 .
\end{aligned}
$$

Let us first look for pure imaginary solutions of this equa- 

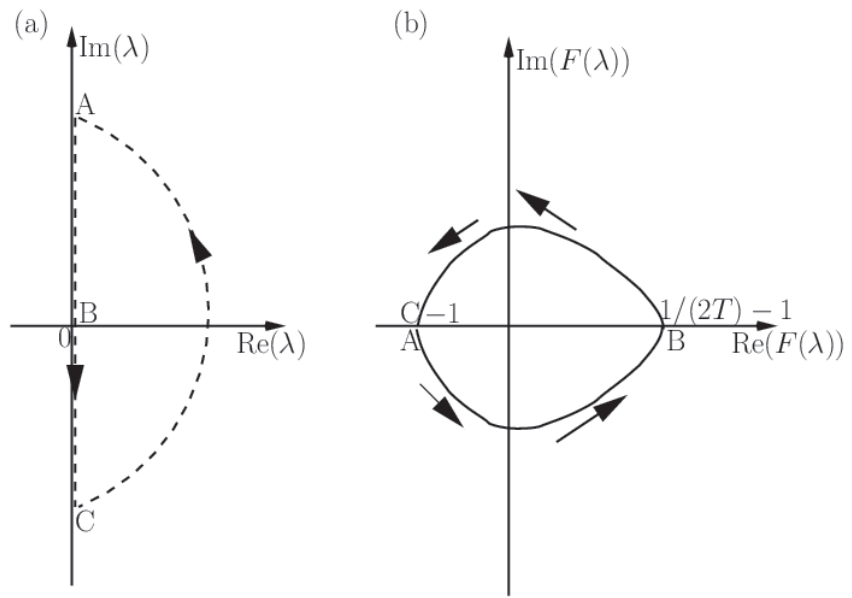

FIG. 5. The loop in the complex $F$-plane, (b), corresponding to the loop in the complex $\lambda$-plane, (a), as determined by the function $F(\lambda)$ in Eq. (33).

tion. Separating into real and imaginary parts, we have

$$
\begin{aligned}
& \operatorname{Re}[F(i \mu ; m, T, \sigma)]=\frac{e^{m T}}{2 T} \sum_{p=0}^{\infty} \frac{(-m T)^{p}}{p !} \\
& \times \int \mathrm{d} \omega g(\omega) \frac{(p+m T)^{2}}{(p+m T)^{2}+(m \sigma \omega+\sqrt{m} \mu)^{2}}-1=0 \\
& \operatorname{Im}[F(i \mu ; m, T, \sigma)]=-\frac{e^{m T}}{2 T} \sum_{p=0}^{\infty} \frac{(-m T)^{p}}{p !} \\
& \times \int \mathrm{d} \omega g(\omega) \frac{(p+m T)(m \sigma \omega+\sqrt{m} \mu)}{(p+m T)^{2}+(m \sigma \omega+\sqrt{m} \mu)^{2}}=0
\end{aligned}
$$

In the second equation above, we make the change of variables $m \sigma \omega+\sqrt{m} \mu=m \sigma x$, and exploit the parity in $x$ of the sum, to obtain

$$
\begin{aligned}
& \operatorname{Im}[F(i \mu ; m, T, \sigma)]= \\
& -m \sigma \int_{0}^{\infty} \mathrm{d} x\left\{\left[g\left(x-\frac{\mu}{\sqrt{m} \sigma}\right)-g\left(-x-\frac{\mu}{\sqrt{m} \sigma}\right)\right]\right. \\
& \left.\times x \sum_{p=0}^{\infty} \frac{(-m T)^{p}}{p !} \frac{p+m T}{(p+m T)^{2}+m^{2} \sigma^{2} x^{2}}\right\}=0
\end{aligned}
$$

It can be shown that the sum on the right-hand side is positive definite for any finite $\sigma$. Furthermore, for our class of distribution functions, one may see that the term in square brackets is positive (respectively, negative) definite for $\mu>0$ (respectively, for $\mu<0$ ). As a consequence, the last equation is never satisfied for $\mu \neq 0$ and finite, and therefore, the eigenvalue equation does not admit pure imaginary solutions [the proof holds also for the particular case $g(\omega)=\delta(\omega)$, as may be checked]. We also conclude that there can be at most one solution with positive real part. In fact, if in the complex $\lambda$-plane, we perform the loop depicted in Fig. 5)(a) (where it is meant that the points $A$ and $C$ represent $\operatorname{Im} \lambda \rightarrow \pm \infty$, respectively, and the radius of the arc extends to $\infty$ ), then, in the complex- $F(\lambda)$ plane, we obtain, due to the sign properties of $\operatorname{Im}[F(i \mu ; m, T, \sigma)]$ just described, the loop qualitatively represented in Fig. [5)(b). The point $F=-1$ in Fig. 5(b) is obtained for $\lambda$, in Fig. 5(a), for values at points $A$ and $C$ and in the whole of the arc extending to infinity. The position of the point $B$ in the complex- $F$ plane is determined by the value of $F(0)$, which is given by

$$
\begin{aligned}
& F(0 ; m, T, \sigma)=\frac{e^{m T}}{2 T} \sum_{p=0}^{\infty} \frac{(-m T)^{p}}{p !} \\
& \int \mathrm{d} \omega g(\omega) \frac{(p+m T)^{2}}{(p+m T)^{2}+(m \sigma \omega)^{2}}-1 .
\end{aligned}
$$

From the well-known theorem of complex analysis on the number of roots of a function in a given domain of the complex plane [29], we therefore obtain that for $F(0 ; m, T, \sigma)>0$, there is one and only one solution of the eigenvalue equation with positive real part; on the other hand, for $F(0 ; m, T, \sigma)<0$, there is no such solution. When the single solution with positive real part exists, it is necessarily real, since a complex solution would imply the existence of its complex conjugate. The value of $F(0 ; m, T, \sigma)$ is readily seen to be equal to $1 /(2 T)-1$ for $\sigma=0$. For positive $\sigma$, the value will depend on the particular form of the distribution function $g(\omega)$. However, it is possible to prove that the value is always smaller than $1 /(2 T)-1$; this is consistent with the physically reasonable fact that if the incoherent state is stable for $\sigma=0$, which happens for $T>1 / 2$, it is all the more stable for $\sigma>0$.

The surface delimiting the region of instability in the $(m, T, \sigma)$ phase space is implicitly defined by Eq. (37) [i.e. $F(0 ; m, T, \sigma)=0$ ], which, in principle, can be solved to obtain the threshold value of $\sigma$ (denoted by $\sigma^{\text {inc }}$ ) as a function of $(m, T): \sigma^{\text {inc }}=\sigma^{\text {inc }}(m, T)$. On physical grounds, we expect that the latter is a single valued function, and that for any given value of $m$, it is a decreasing function of $T$ for $0 \leq T \leq 1 / 2$, reaching 0 for $T=1 / 2$. We are able to prove analytically these facts for the class of unimodal distribution functions $g(\omega)$ considered in this work that includes the Gaussian case. However, we can prove in general for any $g(\omega)$ that $\sigma^{\text {inc }}(m, T)$ tends to 0 for $m \rightarrow \infty$. This is done using the integral representation

$$
\begin{aligned}
& \sum_{p=0}^{\infty} \frac{(-m T)^{p}}{p !} \frac{(p+m T)^{2}}{(p+a)^{2}+(m \sigma \omega)^{2}}=e^{-m T} \\
& -(m \sigma \omega) \int_{0}^{\infty} \mathrm{d} t \exp \left[-m T\left(t+e^{-t}\right)\right] \sin (m \sigma \omega t)
\end{aligned}
$$

For $\sigma>0$ and $m \rightarrow \infty$, one may see that the term within the integral in the last equation tends to $e^{-m T}$. We thus obtain by examining Eq. (37) that $F(0 ; m \rightarrow$ $\infty, T>0, \sigma>0)=-1$. Combined with the fact that $F(0 ; m, T, 0)=1 /(2 T)-1$, this shows that $\sigma^{\text {inc }}(m \rightarrow$ $\infty, 0 \leq T \leq 1 / 2)=0$. 
Let us now turn to the Gaussian case, $g(\omega)=$ $\frac{1}{\sqrt{2 \pi}} \exp \left[-\frac{\omega^{2}}{2}\right]$. Denoting with a subscript $g$ in this case, and using Eq. (38), we have

$$
\begin{aligned}
& F_{g}(0 ; m, T, \sigma)=\frac{1}{2 T}-1-\frac{e^{m T}}{2 T \sqrt{2 \pi}} \int \mathrm{d} \omega e^{-\frac{\omega^{2}}{2}}(m \sigma \omega) \\
& \times \int_{0}^{\infty} \mathrm{d} t \exp \left[-m T\left(t+e^{-t}\right)\right] \sin (m \sigma \omega t)
\end{aligned}
$$

The integral in $\omega$ can be easily performed. Making the change of variable $m \sigma t=y$, we arrive at the following equation:

$$
\begin{aligned}
& F_{g}(0 ; m, T, \sigma)=\frac{1}{2 T}-1 \\
& -\frac{1}{2 T} \int_{0}^{\infty} \mathrm{d} y y e^{-\frac{y^{2}}{2}} \exp \left[m T\left(1-\frac{y}{m \sigma}-e^{-\frac{y}{m \sigma}}\right)\right]
\end{aligned}
$$

The equation $F_{g}(0 ; m, T, \sigma)=0$ defines implicitly the function $\sigma^{\text {inc }}(m, T)$. We can show that this is a singlevalued function with the properties $\frac{\partial \sigma^{\text {inc }}}{\partial m}<0$ and $\frac{\partial \sigma^{\text {inc }}}{\partial T}<$ 0 . We show this by explicitly computing the partial derivatives of $F_{g}(0 ; m, T, \sigma)$ with respect to $m$ and $\sigma$, and by evaluating the behavior with respect to changes in $T$ by adopting a suitable strategy. We begin by computing the derivative with respect to $\sigma$. From Eq. (40), we readily obtain

$$
\begin{aligned}
& \frac{\partial}{\partial \sigma} F_{g}(0 ; m, T, \sigma)=-\frac{1}{2 \sigma^{2}} \int_{0}^{\infty} \mathrm{d} y y^{2} e^{-\frac{y^{2}}{2}}\left(1-e^{-\frac{y}{m \sigma}}\right) \\
& \times \exp \left[m T\left(1-\frac{y}{m \sigma}-e^{-\frac{y}{m \sigma}}\right)\right]
\end{aligned}
$$

which is clearly negative. Second, the derivative with respect to $m$ gives

$$
\begin{aligned}
& \frac{\partial}{\partial m} F_{g}(0 ; m, T, \sigma)=-\frac{1}{2} \int_{0}^{\infty} \mathrm{d} y y e^{-\frac{y^{2}}{2}} \\
& \times\left(1-e^{-\frac{y}{m \sigma}}-\frac{y}{m \sigma} e^{-\frac{y}{m \sigma}}\right) \exp \left[m T\left(1-\frac{y}{m \sigma}-e^{-\frac{y}{m \sigma}}\right)\right] .
\end{aligned}
$$

This derivative is negative, since $1-e^{-x}-x e^{-x}$ is positive for $x>0$. From the implicit function theorems, we then derive that $\frac{\partial \sigma^{\text {inc }}}{\partial m}<0$. The study of the behavior with respect to a change in $T$ is a bit more complicated. Since we are considering $T>0$, we multiply Eq. (40) by $2 T$ to obtain

$$
\begin{aligned}
& 2 T F_{g}(0 ; m, T, \sigma)=1-2 T \\
& -\int_{0}^{\infty} \mathrm{d} y y e^{-\frac{y^{2}}{2}} \exp \left[m T\left(1-\frac{y}{m \sigma}-e^{-\frac{y}{m \sigma}}\right)\right] .
\end{aligned}
$$

Let us consider the integral on the right-hand side

$$
\int_{0}^{\infty} \mathrm{d} y y e^{-\frac{y^{2}}{2}} \exp \left[m T\left(1-\frac{y}{m \sigma}-e^{-\frac{y}{m \sigma}}\right)\right] .
$$

Since $1-x-e^{-x}$ is negative for $x>0$, we conclude that the $T$ derivative of this expression is negative, while its second $T$ derivative is positive. Then the right-hand side of Eq. (43) can be zero, for $T>0$, for at most one value of $T$. Furthermore, since for fixed $y$ and $m$ the value of $y /(m \sigma)$ decreases if $\sigma$ increases, the $T$ value for which $F_{g}(0 ; m, T, \sigma)=0$ decreases for increasing $\sigma$ at fixed $m$. This concludes the proof. Furthermore, for what we have seen before, $\sigma^{\text {inc }}(m, 1 / 2)=0$ and $\lim _{m \rightarrow \infty} \sigma^{\text {inc }}(m, T)=$ 0 for $0 \leq T \leq 1 / 2$.

From the above analysis, it should be clear that the proof is not restricted to the Gaussian case, but would work exactly in the same way for any $g(\omega)$ such that

$$
\beta \int \mathrm{d} x g(x) x \sin (\beta x),
$$

is positive for any $\beta$. However, on physical grounds, we are led to assume that the same conclusions hold for any unimodal $g(\omega)$.

On the basis of our analysis, it follows that at the point of neutral stability, one has $\lambda=0$, which when substituted in Eq. (31) gives $\sigma^{\text {inc }}(m, T)$ to be satisfying

$$
\frac{2 T}{e^{m T}}=\sum_{p=0}^{\infty} \frac{(-m T)^{p}\left(1+\frac{p}{m T}\right)^{2}}{p !} \int_{-\infty}^{\infty} \frac{g(\omega) \mathrm{d} \omega}{\left(1+\frac{p}{m T}\right)^{2}+\frac{\left(\sigma^{\text {inc }}\right)^{2} \omega^{2}}{T^{2}}} .
$$

In the $(m, T, \sigma)$ space, Eq. (46) defines the stability surface $\sigma^{\text {inc }}(m, T)$. There will similarly be the stability surface $\sigma^{\mathrm{coh}}(m, T)$ (see Fig. 1(d) which shows the two surfaces as obtained in $N$-body simulations for $N=500$ for a Gaussian $g(\omega)$ ). The two surfaces coincide on the critical lines on the $(T, \sigma)$ and $(m, T)$ planes where the transition becomes continuous; outside these planes, the surfaces enclose the first-order transition surface $\sigma_{c}(m, T)$ i.e., $\sigma^{\mathrm{coh}}(m, T)>\sigma_{c}(m, T)>\sigma^{\text {inc }}(m, T)$. We now show by taking limits that the surface $\sigma^{\text {inc }}(m, T)$ meets the critical lines on the $(T, \sigma)$ and $(m, T)$ planes, and also obtain its intersection with the $(m, \sigma)$-plane. On considering $m \rightarrow 0$ at a fixed $T$, only the $p=0$ term in the sum in Eq. (46) contributes, giving

$$
\lim _{m \rightarrow 0, T \text { fixed }} \sigma^{\text {inc }}(m, T)=\sigma_{c}(m=0, T),
$$

with the implicit expression of $\sigma_{c}(m=0, T)$ given by Eq. (17). Similarly, one finds that

$$
\lim _{T \rightarrow T_{c}^{-}, m \text { fixed }} \sigma^{\text {inc }}(m, T)=0,
$$

that is, on the $(m, T)$ plane, the transition line is given by $T_{c}=1 / 2$. When $T \rightarrow 0$ at a fixed $m$, we get

$$
\sigma_{\text {noiseless }}^{\text {inc }}(m) \equiv \lim _{T \rightarrow 0, m \text { fixed }} \sigma^{\text {inc }}(m, T),
$$

with

$$
1=\frac{\pi g(0)}{2 \sigma_{\text {noiseless }}^{\text {inc }}}-\frac{m}{2} \int_{-\infty}^{\infty} \frac{g(\omega) \mathrm{d} \omega}{1+m^{2}\left(\sigma_{\text {noiseless }}^{\text {inc }}\right)^{2} \omega^{2}} .
$$

For the case of a Gaussian $g(\omega)$, the limits (47) and (48) are shown in Figs. 1(b) and 1(c), while the limit Eq. (49) is shown in Fig. 6, 


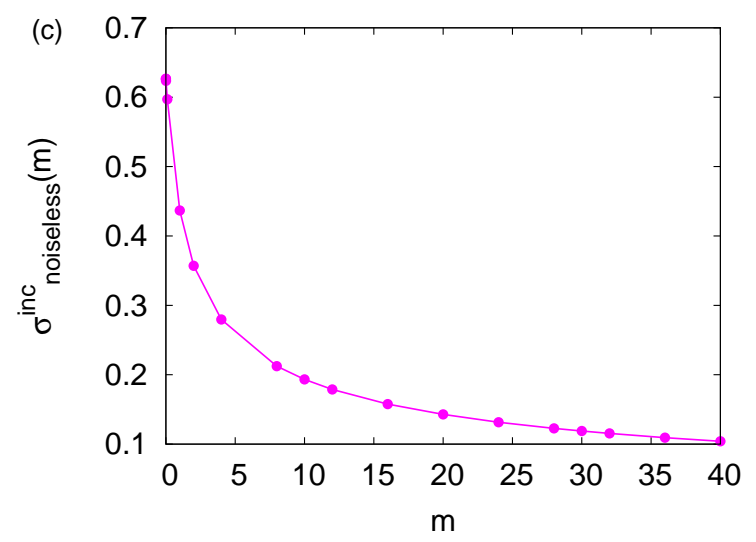

FIG. 6. (Color online) The figure shows the limit Eq. (49) for the case of a Gaussian $g(\omega)$ with zero mean and unit width.

\section{COMPARISON WITH NUMERICS}

For a Gaussian $g(\omega)$, Eq. (46) gives

$$
1=\frac{e^{m T} \sqrt{\pi}}{2 \sqrt{2} \sigma^{\text {inc }}} \sum_{p=0}^{\infty} \frac{(-m T)^{p}\left(1+\frac{p}{m T}\right)}{p ! e^{-\frac{T^{2}(1+p / m T)^{2}}{2\left(\sigma^{\text {inc }}\right)^{2}}}} \operatorname{Erfc}\left[\frac{T\left(1+\frac{p}{m T}\right)}{\sigma^{\text {inc } \sqrt{2}}}\right] .
$$

Choosing $m=20$ and $T=0.25$, the above equation gives $\sigma^{\text {inc }}(m, T) \approx 0.10076$. Then, preparing the system in the incoherent stationary state at a given $\sigma$, our theoretical analysis predicts that $r$, in the dynamically unstable regime of the incoherent state (i.e., with $\sigma<\sigma^{\text {inc }}$ ), relaxes at long times to its steady-state value corresponding to the synchronized phase. For $\sigma>\sigma^{\text {inc }}(m, T)$, when the incoherent initial state is linearly stable, $r$ is zero for all times. We now compare the above continuum-limit predictions with $N$-body simulations. We monitor the evolution of $r$ in time while starting from the incoherent stationary state. To discuss the results, we employ the standard picture of phase transitions occurring dynamically as the dissipative relaxation of the order parameter towards the minimum of a phenomenological Landau free energy [30]. For a first-order phase transition, we draw in Fig. 7 the corresponding schematic free energy $F(r)$ versus $r$ for fixed $m$ and $T$ at different $\sigma$ 's [31]. The picture helps to explain, e.g., the flips in $r$ in Fig. 4 which correspond to dynamics at $\sigma$ close to $\sigma_{c}$, when the system switches back and forth between the two almost stable synchronized and incoherent states.

Let us investigate the dynamics for $\sigma$ around $\sigma^{\text {inc }}(m, T)$. Figures 8 (a)-(d) show simulation results for $r$ versus time for four values of $\sigma$, two below and two above $\sigma^{\text {inc }}(m, T)$. In each case, we display the dependence for 20 realizations of the initial incoherent state for three values of $N$. Figure 8 (a) for $\sigma<\sigma^{\text {inc }}(m, T)$ illustrates that the system while starting from the unstable incoherent state settles down in time into the globally stable synchronized state; this corresponds to dynamics in the landscape in Fig. 7(i). The relaxation of $r$ from the ini-

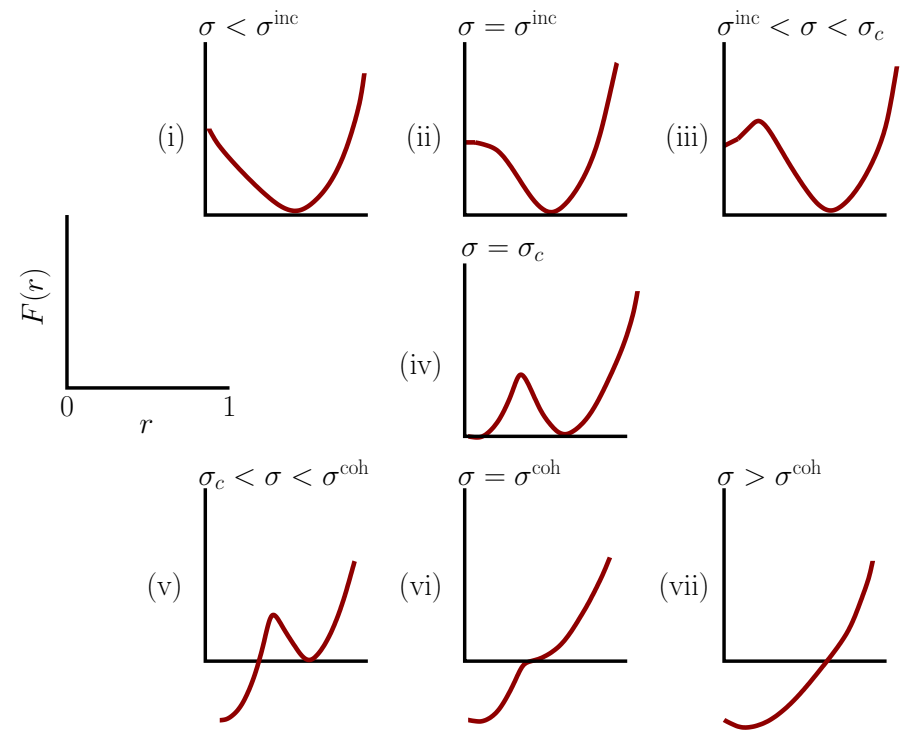

FIG. 7. (Color online) Schematic Landau free energy $F(r)$ versus $r$ for first-order transitions at fixed $m$ and $T$ while varying $\sigma$. Panels (i) and (vii) correspond to the synchronized and incoherent phase being at the global minimum. In panel (iii) (respectively, (v)), the synchronized (respectively, incoherent) phase is at the global minimum, while the incoherent (respectively, synchronized) phase is at a local minimum, hence, metastable. Panel (iv) corresponds to the first-order transition point, with the two phases coexisting at two minima of equal heights.

tial to final synchronized state value occurs exponentially fast in time as $e^{\lambda t}$; the growth rate $\lambda$ is obtained from Eq. (31) after substituting a Gaussian distribution for $g(\omega)$. In Fig. 9 we demonstrate a match of $\lambda$ in theory and simulations.

In Fig. 8(b), when $\sigma$ is larger than in Fig. $8(a)$, yet below $\sigma^{\text {inc }}(m, T)$, the system settles at long times into the synchronized state for all realizations. Yet, some of them, at short times, tend to stay in the initial incoherent state due to finite- $N$ effects not captured by our continuum limit theory; see Eq. (27). For $\sigma>\sigma^{\text {inc }}(m, T)$, but $\sigma<\sigma_{c}(m, T)$, we expect on the basis of the landscape sketched in Fig. [7(iii) that the system settles at long times into the globally stable synchronized state, while for finite times, remains trapped in the metastable incoherent state. Indeed, Fig. 8(c) shows that most realizations relax to synchronized states. However, as $N$ increases, the number of realizations staying close to the initial incoherent state for a finite time increases. We found that the fraction $\eta$ of realizations relaxing to synchronized state within a fixed time decreases exponentially fast in $N$ for large $N$; see Fig. 10. This observation implies that for the fixed time of observation, there exists a larger $N$ than the ones in Fig. 8(c) for which all realizations remain close to the incoherent state; it then follows that in the continuum limit, all realizations stay close to the incoherent state.

To explain the above mentioned behavior of $\eta$ with $N$, 
(a)

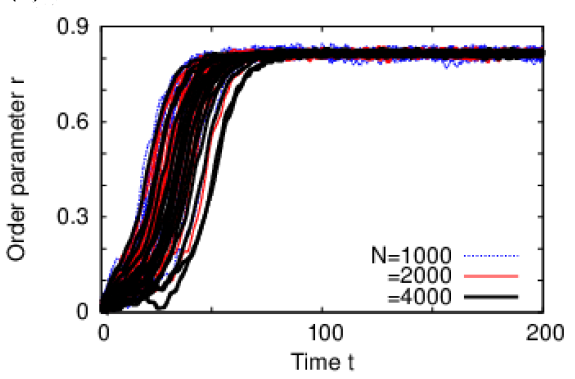

(b)

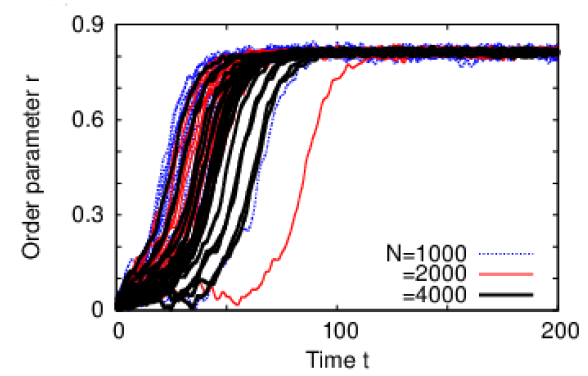

(c)

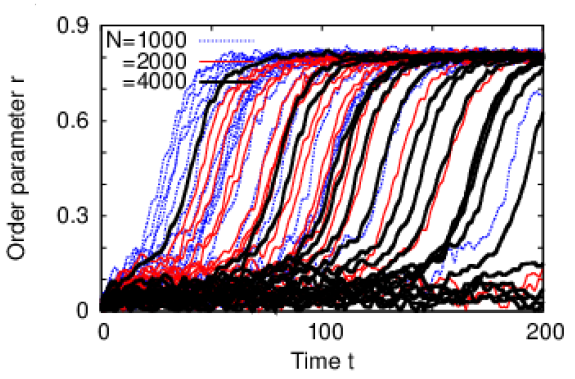

(d).

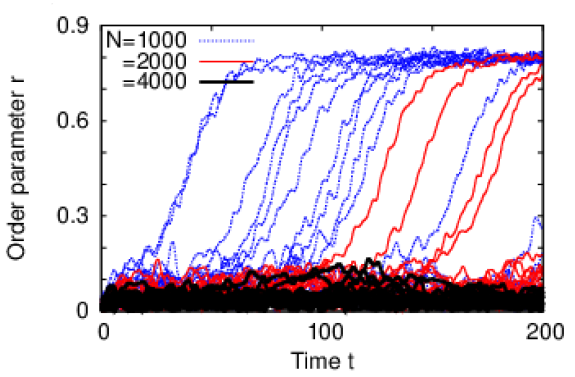

FIG. 8. (Color online) Panels (a)-(d) show $r$ vs. time at $m=$ $20, T=0.25$ for four values of $\sigma$, two below ((a): $\sigma=0.09$, (b): $\sigma=0.095)$, and two above ((c): $\sigma=0.11,(\mathrm{~d}): \sigma=0.12)$ the theoretical threshold $\sigma^{\text {inc }}(m, T) \approx 0.10076$. The data are obtained in $N$-body simulations for a Gaussian $g(\omega)$ with zero mean and unit width.

let us first consider the noisy dynamics of a single particle on a potential landscape, when the typical time to get out of a metastable state is given in the weak-noise limit by the Kramers time, i.e., an exponential in the ratio of the potential energy barrier to come out of the metastable state to the strength of the noise 32]. For the dynamics of the order parameter on a free-energy landscape for mean-field systems, the escape time out of a metastable state obeys Kramers formula with the value of the freeenergy barrier replacing the potential energy barrier, and with an extra factor of $N$ multiplying the barrier height [33]; this explains Fig. 8(c) and the behavior of $\eta$.

Figure 8(d), for $\sigma$ larger than $\sigma^{\text {inc }}(m, T)$ than in Fig. 8(c), shows that with respect to (c), more realizations stay close to the initial incoherent state for longer times, due to a larger barrier between the incoherent and synchronized state. On the basis of the above discussions, we conclude that our theoretical predictions are borne out by our simulation results. In particular, the simulation results for $N=500$ suggest that the stability threshold of the incoherent state lies in between $\sigma=0.095$ and $\sigma=0.11$, a range that includes its theoretical continuumlimit value $(\approx 0.10076)$.
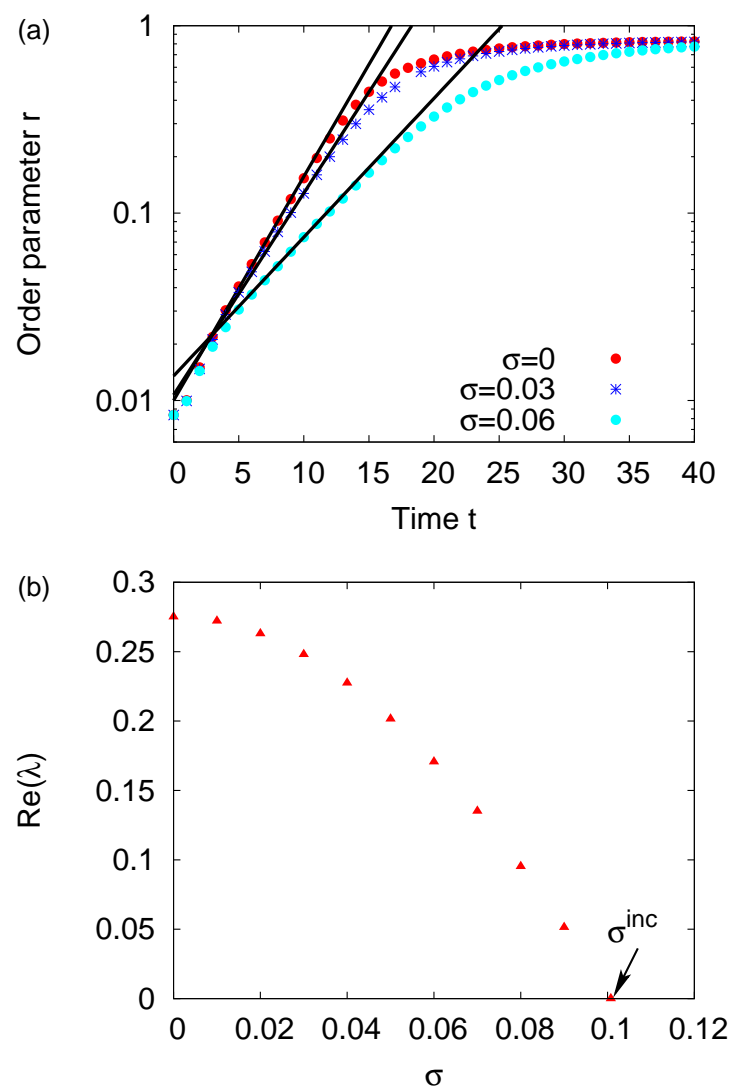

FIG. 9. (Color online) (a) Simulation results denoted by points, demonstrating exponentially fast relaxation $\sim e^{\lambda t}$ of $r$ from its initial incoherent state value to its final synchronized state value for values of $\sigma$ below $\sigma^{\text {inc }}(m, T) \approx 0.10076$ for a Gaussian $g(\omega)$ with $m=20, T=0.25, N=10^{4}$; the black solid lines denote exponential growth with theoretically computed growth rates $\lambda$ obtained from Eq. (31) for a Gaussian $g(\omega)$ with zero mean and unit width. The simulation data are obtained from $N$-body simulation for a Gaussian $g(\omega)$ with zero mean and unit width. (b) Theoretical $\lambda$ as a function of $\sigma$ for the same $m$ and $T$ values; in particular, $\lambda$ hits zero at the stability threshold $\sigma^{\text {inc }}(m, T)$. 


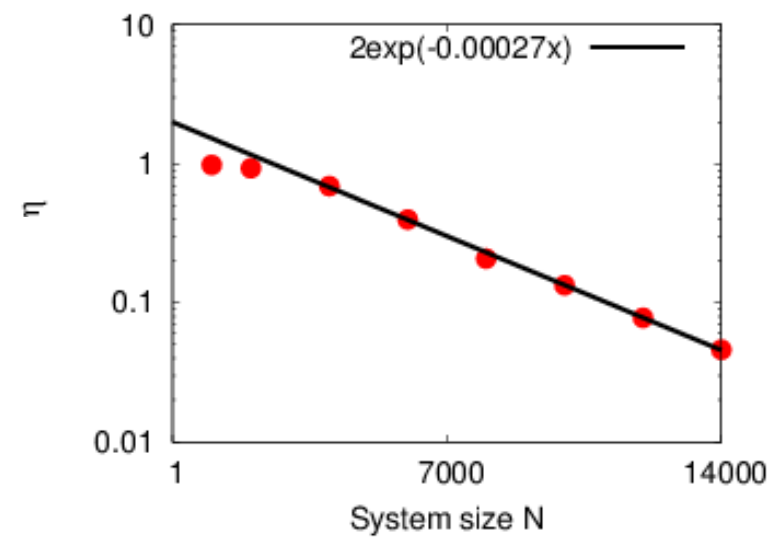

FIG. 10. (Color online) For $m=20, T=0.25, \sigma=0.11$, the figure shows the fraction $\eta$ of realizations of initial incoherent state relaxing to synchronized state within the fixed time of observation $t=200$, for a value of $\sigma$ above $\sigma^{\text {inc }}(m, T)$, for which the incoherent phase is linearly stable in the continuum limit. The figure shows that $\eta$ for large $N$ decreases exponentially fast with increase of $N$. The data are obtained in $N$-body simulations for a Gaussian $g(\omega)$ with zero mean and unit width.

\section{CONCLUSIONS}

To summarize, we considered an extension of the Kuramoto model that includes an inertial term and a stochastic noise. For a general unimodal frequency distribution, we obtained the complete phase diagram of the model, demarcating parameter ranges to observe synchronization. We showed that the system displays a nonequilibrium first-order transition from a synchronized phase at low parameter values to an incoherent phase at high values. The phase diagram contains all previous results derived in specific limits of the dynamics. While we provided strong numerical evidence for the existence of both the synchronized and the incoherent phase, only the latter could be treated analytically to obtain the corresponding linear stability threshold that bounds the firstorder transition point from below. It would be interesting to consider possible extension of our studies to systems with non-mean-field couplings, taking hints from similar previous studies in specific limits of the dynamics [34 37].

We acknowledge fruitful discussions with T. Dauxois, D. Mukamel, C. Nardini, A. Patelli and H. Touchette, support of ENS-Lyon and the grants CEFIPRA 4604-3 and ANR-10-CEXC-010-01.

\section{Appendix A: Proof that the dynamics Eq. (12) does not satisfy detailed balance}

In this section, we prove that the dynamics Eq. (12) does not satisfy detailed balance unless $g(\omega)=\delta(\omega)$, thus $\sigma$ is zero. For simplicity, we discuss the proof here for the case of two distinct natural frequencies [bimodal $g(\omega)$ ].
Let us say that in a given realization of $g(\omega)$, there are $N_{1}$ oscillators with natural frequencies $\omega_{1}$, and $N_{2}$ oscillators with natural frequencies $\omega_{2}$, where $N_{1}+N_{2}=N$.

To prove that the dynamics Eq. (12) does not satisfy detailed balance unless $\sigma=0$, we rewrite the FokkerPlanck Eq. (20) as

$$
\begin{aligned}
& \frac{\partial f_{N}(\mathbf{x})}{\partial t}=-\sum_{i=1}^{2 N} \frac{\partial\left(A_{i}(\mathbf{x}) f_{N}(\mathbf{x})\right)}{\partial x_{i}} \\
& +\frac{1}{2} \sum_{i, j=1}^{2 N} \frac{\partial^{2}\left(B_{i, j}(\mathbf{x}) f_{N}(\mathbf{x})\right)}{\partial x_{i} \partial x_{j}}
\end{aligned}
$$

where

$$
x_{i}=\left\{\begin{array}{l}
\theta_{i} ; i=1,2, \ldots, N \\
v_{i-N} ; i=N+1, \ldots, 2 N
\end{array}\right.
$$

and

$$
\mathbf{x}=\left\{x_{i}\right\}_{1 \leq i \leq 2 N} .
$$

In Eq. (A1), the drift vector $A_{i}(\mathbf{x})$ is given by

$$
A_{i}(\mathbf{x})=\left\{\begin{array}{l}
v_{i} ; i=1,2, \ldots, N \\
-\frac{1}{\sqrt{m}} v_{i-N}+\frac{1}{N} \sum_{j=1}^{N} \sin \left(\theta_{j}-\theta_{i-N}\right) \\
+\sigma\left(\Omega^{T}\right)_{i-N} ; i=N+1, \ldots, 2 N
\end{array}\right.
$$

while the diffusion matrix is

$$
B_{i, j}(\mathbf{x})=\left\{\begin{array}{l}
\frac{2 T}{\sqrt{m}} \delta_{i j} ; i, j>N \\
0, \text { Otherwise. }
\end{array}\right.
$$

The dynamics described by the Fokker-Planck equation of the form Eq. (A1) satisfies detailed balance if and only if the following conditions are satisfied [38]:

$$
\begin{aligned}
& \epsilon_{i} \epsilon_{j} B_{i, j}(\epsilon \mathbf{x})=B_{i, j}(\mathbf{x}) \\
& \epsilon_{i} A_{i}(\epsilon \mathbf{x}) f_{N}^{s}(\mathbf{x})=-A_{i}(\mathbf{x}) f_{N}^{s}(\mathbf{x})+\sum_{j=1}^{2 N} \frac{\partial B_{i, j}(\mathbf{x}) f_{N}^{s}(\mathbf{x})}{\partial x_{j}}
\end{aligned}
$$

where $f_{N}^{s}(\mathbf{x})$ is the stationary solution of Eq. (A1). Here, $\epsilon_{i}= \pm 1$ is a constant that denotes the parity with respect to time reversal of the variables $x_{i} \mathrm{~s}$ : Under time reversal, the latter transform as $x_{i} \rightarrow \epsilon_{i} x_{i}$, where $\epsilon_{i}=-1$ or +1 depending on whether $x_{i}$ is odd or even under time reversal. In our case, $\theta_{i}$ s are even, while $v_{i}$ s are odd.

Using Eq. A5, we see that the condition Eq. A6 is trivially satisfied for our model. To check the other condition, we formally solve Eq. (A7) for $f_{N}^{s}(\mathbf{x})$ and check if the solution solves Eq. A1 in the stationary state. From Eq. (A7), we see that for $i=1,2, \ldots, N$, the condition reduces to

$$
\epsilon_{i} A_{i}(\epsilon \mathbf{x}) f_{N}^{s}(\mathbf{x})=-A_{i}(\mathbf{x}) f_{N}^{s}(\mathbf{x}),
$$

which, using Eq. A4 , is obviously satisfied. For $i=$ $N+1, \ldots, 2 N$, we have

$$
v_{k} f_{N}^{s}(\mathbf{x})=-\frac{T \partial f_{N}^{s}(\mathbf{x})}{\partial v_{k}} ; k=i-N
$$


Solving Eq. (A99), we get

$$
f_{N}^{s}(\mathbf{x}) \propto d\left(\theta_{1}, \theta_{2}, \ldots, \theta_{N}\right) \exp \left[-\frac{1}{2 T} \sum_{k=1}^{N} v_{k}^{2}\right]
$$

where $d\left(\theta_{1}, \theta_{2}, \ldots, \theta_{N}\right)$ is a yet undetermined function. Substituting Eq. A10 into Eq. (A1), and requiring that it is a stationary solution, we get that $\sigma$ has to be equal to zero and that $d\left(\theta_{1}, \theta_{2}, \ldots, \theta_{N}\right)=\exp (-$ $\left.\frac{1}{2 N T} \sum_{i, j=1}^{N}\left[1-\cos \left(\theta_{i}-\theta_{j}\right)\right]\right)$. Thus, for $\sigma=0$, when the dynamics reduces to that of the BMF model, we get the stationary solution as

$$
f_{N, \sigma=0}^{s}(\mathbf{z}) \propto \exp \left[-\frac{H}{T}\right]
$$

where $H$ is the Hamiltonian (expressed in terms of dimensionless variables introduced above). The lack of detailed balance for $\sigma \neq 0$ obviously extends to any distribution $g(\omega)$.

\section{Appendix B: Simulation details}

Here we describe the method to simulate the dynamics Eq. (12) for given values of $m, T, \sigma$ (note that we are dropping overbars for simplicity of notation), and for a given realization of $\omega_{i}$ 's, by employing a numerical integration scheme [39]. To simulate the dynamics over a time interval $[0: \mathcal{T}]$, we first choose a time step size $\Delta t \ll 1$. Next, we set $t_{n}=n \Delta t$ as the $n$-th time step of the dynamics, where $n=0,1,2, \ldots, N_{t}$, and $N_{t}=\mathcal{T} / \Delta t$. In the numerical scheme, we first discard at every time step the effect of the noise (i.e., consider $1 / \sqrt{m}=0$ ), and employ a fourth-order symplectic algorithm to integrate the resulting symplectic part of the dynamics [40]. Following this, we add the effect of noise, and implement an Euler-like first-order algorithm to update the dynamical variables. Specifically, one step of the scheme from $t_{n}$ to $t_{n+1}=t_{n}+\Delta t$ involves the following updates of the dynamical variables for $i=1,2, \ldots, N$ : For the symplectic part, we have, for $k=1, \ldots, 4$,

$v_{i}\left(t_{n}+\frac{k \Delta t}{4}\right)=v_{i}\left(t_{n}+\frac{(k-1) \Delta t}{4}\right)+b(k) \Delta t\left[r\left(t_{n}+\frac{(k-1) \Delta t}{4}\right) \sin \left\{\psi\left(t_{n}+\frac{(k-1) \Delta t}{4}\right)-\theta_{i}\left(t_{n}+\frac{(k-1) \Delta t}{4}\right)\right\}+\sigma \omega_{i}\right] ;$

$r\left(t_{n}+\frac{(k-1) \Delta t}{4}\right)=\sqrt{r_{x}^{2}+r_{y}^{2}}, \quad \psi\left(t_{n}+\frac{(k-1) \Delta t}{4}\right)=\tan ^{-1} \frac{r_{y}}{r_{x}}$,

$r_{x}=\frac{1}{N} \sum_{j=1}^{N} \sin \left[\theta_{j}\left(t_{n}+\frac{(k-1) \Delta t}{4}\right)\right], \quad r_{y}=\frac{1}{N} \sum_{j=1}^{N} \cos \left[\theta_{j}\left(t_{n}+\frac{(k-1) \Delta t}{4}\right)\right]$,

$\theta_{i}\left(t_{n}+\frac{k \Delta t}{4}\right)=\theta_{i}\left(t_{n}+\frac{(k-1) \Delta t}{4}\right)+a(k) \Delta t v_{i}\left(t_{n}+\frac{k \Delta t}{4}\right)$

where the constants $a(k)$ 's and $b(k)$ 's are obtained from Ref. [40]: one has

$$
\begin{array}{ll}
a(1)=0.5153528374311229364, & a(2)=-0.085782019412973646, \\
a(3)=0.4415830236164665242, & a(4)=0.1288461583653841854, \\
b(1)=0.1344961992774310892, & b(2)=-0.2248198030794208058, \\
b(3)=0.7563200005156682911, & b(4)=0.3340036032863214255 .
\end{array}
$$

At the end of the updates Eqs. (B1) and (B2), we have the set $\left\{\theta_{i}\left(t_{n+1}\right), v_{i}\left(t_{n+1}\right)\right\}$. Next, we include the effect of the stochastic noise by keeping $\theta_{i}\left(t_{n+1}\right)$ 's unchanged, but by updating $v_{i}\left(t_{n+1}\right)$ 's as

$v_{i}\left(t_{n+1}\right) \rightarrow v_{i}\left(t_{n+1}\right)\left[1-\frac{1}{\sqrt{m}} \Delta t\right]+\sqrt{2 \Delta t \frac{T}{\sqrt{m}}} \Delta X\left(t_{n+1}\right)$.
Here $\Delta X$ is a Gaussian distributed random number with zero mean and unit variance.
[1] M. Bier, B. M. Bakker, and H. V. Westerhoff, Biophys J. 78, 1087 (2000).
[2] A. T. Winfree, The Geometry of Biological Time (Springer, New York, 1980) 
[3] J. Buck, Quart. Rev. Biol. 63, 265 (1988).

[4] K. Wiesenfeld, P. Colet, and S. H. Strogatz, Phys. Rev. E. 57, 1563 (1998).

[5] G. Filatrella, A. H. Nielsen, and N. F. Pedersen, Eur. Phys. J B 61, 485 (2008).

[6] M. Rohden, A. Sorge, M. Timme, and D. Witthaut, Phys. Rev. Lett. 109, 064101 (2012).

[7] F. Dörfler, M. Chertkov, and F. Bullo, Proc. Natl. Acad. Sci. USA 110, 2005 (2013).

[8] Z. Néda, E. Ravasz, T. Vicsek, Y. Brechet, and A. L. Barabási, Phys. Rev. E 61, 6987 (2000).

[9] S. Y. Ha, E. Jeong, and M. J. Kang, Nonlinearity 23, 3139 (2010).

[10] S. H. Strogatz, Sync: The emerging science of spontaneous order (Hyperion, New York, 2003).

[11] Y. Kuramoto, Chemical oscillations, Waves and Turbulence (Springer, Berlin, 1984).

[12] S. H. Strogatz, Physica D 143, 1 (2000); J. A. Acebrón, L. L. Bonilla, C. J. Pérez Vicente, Félix Ritort, and R. Spigler, Rev. Mod. Phys. 77, 137 (2005).

[13] J. A. Acebrón, and R. Spigler, Phys. Rev. Lett. 81, 2229 (1998).

[14] H. Hong, M. Y. Choi, B-G. Yoonk, K. Park, and K-S. Soh, J. Phys. A: Math. Gen. 32, L9 (1999).

[15] J. A. Acebrón, L. L. Bonilla, and R. Spigler, Phys. Rev. E 62, 3437 (2000).

[16] H. Sakaguchi, Prog. Theor. Phys. 79, 39 (1988).

[17] B. Ermentrout, J. Math. Biol. 29, 571 (1991).

[18] S. Inagaki, Prog. Theor. Phys. 90, 577 (1993); M. Antoni and S. Ruffo, Phys. Rev. E 52, 2361 (1995).

[19] K. Mallick, Pramana - J. Phys. 73, 417 (2009).

[20] Nonequilibrium Statistical Mechanics in One Dimension, edited by V. Privman (Cambridge University Press, Cambridge, 1997).

[21] A. Campa, T. Dauxois, and S. Ruffo, Phys. Rep. 480, 57 (2009).

[22] P. H. Chavanis, e-print arXiv:1306.1203
[23] H. A. Tanaka, A. J. Lichtenberg, and S. Oishi, Phys. Rev. Lett. 78, 2104 (1997).

[24] We take the width of a Gaussian distribution to refer to its standard deviation.

[25] H. Touchette, Phys. Rep. 478, 1 (2009).

[26] B. Derrida, Pramana - J. Phys 64, 695 (2005).

[27] N. Goldenfeld, Lectures on phase transitions and the renormalization group (Addison-Wesley, Advanced Book Program, 1992).

[28] K. Huang, Statistical mechanics (Wiley, New York, 1987).

[29] V. I. Smirnov, A course of higher mathematics. Vol. 3. Part. 2, Complex variables special functions (Pergamon Press, Oxford, 1964); chapter 1, section 22.

[30] K. Binder, Rep. Prog. Phys. 50, 783 (1987).

[31] For non-zero $\sigma$, one should draw landscapes for the freeenergy-like large deviation functional; we assume here the landscape picture of phase transitions to also hold for this quantity.

[32] H. A. Kramers, Physica 7, 284 (1940).

[33] R. B. Griffiths, C. Y. Weng, and J. S. Langer, Phys. Rev. 149, 301 (1966).

[34] A. Campa, A. Giansanti, and D. Moroni, J. Phys. A: Math. Gen. 36, 6897 (2003).

[35] R. Bachelard, T. Dauxois, G. De Ninno, S. Ruffo and F. Staniscia, Phys. Rev. E 83, 061132 (2011).

[36] S. Gupta, M. Potters, and S. Ruffo, Phys. Rev. E 85, 066201 (2012).

[37] S. Gupta, A. Campa, and S. Ruffo, Phys. Rev. E 86, 061130 (2012).

[38] C. W. Gardiner, Handbook of Stochastic Methods for Physics, Chemistry and the Natural Sciences (Springer, Berlin, 1983).

[39] C. Nardini, S. Gupta, S. Ruffo, T. Dauxois, and F. Bouchet, J. Stat. Mech.: Theory Exp. P12010 (2012).

[40] R. I. McLachlan and P. Atela, Nonlinearity 5, 541 (1992). 